\title{
The Fuzzy Stability of a Pexiderized Functional Equation
}

\author{
Nabin Chandra Kayal, Pratap Mondal and T.K. Samanta
}

Abstract. In this paper, Hyers-Ulam-Rassias Stability of the Pexiderized functional equation $f(x+y)=g(x)+h(y)$ is concerned in fuzzy Banach spaces.

\section{INTRODUCTION}

In 1940 stability problem of a functional equation was initiated by Ulam [14] concerning the stability of group homomorphism. In the next year, Hyers [6] gave answer for Cauchy functional equation in Banach spaces. T. Aoki [15] and Th. M. Rassias [16] generalized Hyers's theorem for additive mappings and linear mappings by considering an unbounded Cauchy difference respectively. Gavruta [10] generalized Rassias theorem by replacing the unbounded Cauchy difference by a general control function in the spirit of Rassias's approach. F. Skof [7] generalized Hyers-Ulam stability theorem for the function $f: X \rightarrow Y$, where $X$ is a normed linear space and $Y$ is a Banach space. Afterwards, the result of Skof was extended by P. W. Cholewa [11] and S. Czerwik [13].

Fuzzy set theory was initiated by Zadeh [8] and after the introduction of the notion of fuzzy norm on a linear space by Katsaras [2], many authors $[12,17]$, gave various ideas of fuzzy norm. Thereafter various notions of Banach spaces have been generalized in fuzzy Banach spaces. In fact, the notion of the Hyers-Ulam-Rassias stability for various functional equations are being generalized in fuzzy Banach Spaces by several authors $[3,5,9,18,19]$.

In this paper, we investigate the generalized Hyers-Ulam-Rassias stability for the functional equation $f(x+y)=g(x)+h(y)$ in fuzzy Banach spaces.

\section{Preliminaries}

In the sequel, we need some definitions which are given bellow.

2010 Mathematics Subject Classification. Primary: 03E72, 97I70; Secondary 39B82.

Key words and phrases. Fuzzy norm, fuzzy Banach space, Pexiderized functional equation, Hyers-Ulam-Rassias stability. 
Definition $2.1([4])$. A binary operation $*:[0,1] \times[0,1] \longrightarrow[0,1]$ is continuous $t$ - norm if $*$ satisfies the following conditions:

(i) $*$ is commutative and associative;

(ii) $*$ is continuous;

(iii) $a * 1=a \quad \forall a \in[0,1]$;

(iv) $a * b \leq c * d$ whenever $a \leq c, b \leq d$ and $a, b, c, d \in[0,1]$.

Again we assume that $a * a=a \forall a \in[0,1]$.

Definition 2.2 ([17]). The 3 - tuple $(X, N, *)$ is called a fuzzy normed linear space if $X$ is a real linear space, $*$ is a continuous $\mathrm{t}$ - norm and $N$ is a fuzzy set in $X \times(0, \infty)$ satisfying the following conditions:

(i) $N(x, t)>0$,

(ii) $N(x, t)=1$ if and only if $x=0$,

(iii) $N(c x, t)=N\left(x, \frac{t}{|c|}\right)$ if $c \neq 0$,

(iv) $N(x, s) * N(y, t) \leqslant N(x+y, s+t)$,

(v) $N(x, \cdot):(0, \infty) \rightarrow(0,1]$ is continuous

for all $x, y \in X$ and $t, s>0$.

Note that $N(x, t)$ can be thought of as the degree of nearness between $x$ and null vector 0 with respect to $t$.

Example 2.1. Let $X=[0, \infty), a * b=a b$, for every $a, b \in[0,1]$, and $\|\cdot\|$ be the usual metric defined on $X$. Define $N(x, t)=e^{-\frac{\|x\|}{t}}$ for all $x \in X$. Then clearly $(X, N, *)$ is a fuzzy normed linear space.

Example 2.2. Let $(X,\|\cdot\|)$ be a normed linear space, and let $a * b=a b$ or $a * b=\min \{a, b\}$ for all $a, b \in[0,1]$. Let $N(x, t)=\frac{t}{t+\|x\|}$ for all $x \in X$ and $t>0$. Then $(X, N, *)$ is a fuzzy normed linear space and this fuzzy norm $N$ induced by $\|\cdot\|$ is called the standard fuzzy norm.

Note 2.1. According to George and Veeramani [1], it can be proved that every fuzzy normed linear space is a metrizable topological space. In fact, also it can be proved that if $(X,\|\cdot\|)$ is a normed linear space, then the topology generated by $\|\cdot\|$ coincides with the topology generated by the fuzzy norm $N$ of Example 2.2. As a result, we can say that an ordinary normed linear space is a special case of fuzzy normed linear space.

Remark 2.1. In fuzzy normed linear space $(X, N, *)$, for all $x \in X, N(x, \cdot)$ is non-decreasing with respect to the variable $t$ and $\lim _{t \rightarrow \infty} N(x, t)=1$.

Definition 2.3. [17] Let $(X, N, *)$ be a fuzzy normed linear space. A sequence $\left\{x_{n}\right\}$ in $X$ is said to be convergent or converge if there exists an $x \in X$ such that $\lim _{n \rightarrow \infty} N\left(x_{n}-x, t\right)=1$. In this case, $x$ is called the limit of the sequence $\left\{x_{n}\right\}$ and we denote it by $N-\lim _{n \rightarrow \infty} x_{n}=x$. 
Definition 2.4. [17] Let $(X, N, *)$ be a fuzzy normed linear space. A sequence $\left\{x_{n}\right\}$ in $X$ is called Cauchy sequence if for each $\varepsilon>0$ and $t>0$ there exists an $n_{0} \in \mathbb{N}$ such that for all $n \geqslant n_{0}$ and all $p>0$, we have $N\left(x_{n+p}-x_{n}, t\right)>1-\varepsilon$.

\section{Stability of The Functional Equation}

Throughout this section, $X$ is assumed to be a real vector space and $(Y, N)$ is assumed to be a fuzzy Banach space.

Theorem 3.1. Let $\phi: X^{2} \rightarrow \mathbb{R}^{+}$be a mapping such that

$$
\widetilde{\phi}(x, y):=\sum_{i=0}^{\infty} \frac{\phi\left(3^{i} x, 3^{i} y\right)}{3^{i}}<\infty, \quad \text { for all } x, y \in X .
$$

Let $f, g, h: X \rightarrow Y$ be mappings such that

$$
\lim _{t \rightarrow \infty} N(f(x+y)-g(x)-h(x), t \phi(x, y))=1
$$

uniformly on $X^{2}$. Then there exists a unique mapping $A: X \rightarrow Y$ such that

$$
A(x+y)=A(x)+A(y)
$$

for all $x, y \in X$ and if for some $\delta>0, \alpha>0$

$$
N(f(x+y)-g(x)-h(y), \delta \phi(x, y)) \geq \alpha
$$

for all $x, y \in X$, then

$$
\begin{aligned}
N(f(x)- & A(x)-f(0), \frac{\delta}{3}\left[\widetilde{\phi}\left(\frac{x}{2}, \frac{-x}{2}\right)+\right. \\
& \widetilde{\phi}\left(\frac{-x}{2}, \frac{x}{2}\right)+\widetilde{\phi}\left(\frac{x}{2}, \frac{x}{2}\right)+2 \widetilde{\phi}\left(\frac{-x}{2}, \frac{-x}{2}\right)+ \\
& \left.\left.\widetilde{\phi}\left(\frac{-x}{2}, \frac{3 x}{2}\right)+\widetilde{\phi}\left(\frac{3 x}{2}, \frac{-x}{2}\right)+\widetilde{\phi}\left(\frac{3 x}{2}, \frac{3 x}{2}\right)\right]\right) \geq \alpha
\end{aligned}
$$

and

$$
N-\lim _{n \rightarrow \infty} \frac{f\left(3^{n} x\right)}{3^{n}}=N-\lim _{n \rightarrow \infty} \frac{g\left(3^{n} x\right)}{3^{n}}=N-\lim _{n \rightarrow \infty} \frac{h\left(3^{n} x\right)}{3^{n}}=A(x)
$$

for all $x \in X$.

Proof. Corresponding to a given $\epsilon>0$ and (3.1), there exists some $t_{0}>0$ such that

$$
N(f(x+y)-g(x)-h(y), t \phi(x, y)) \geq 1-\epsilon
$$

for all $x, y \in X$ and $t \geq t_{0}$. Let

$$
\phi_{1}(x, y)=\phi\left(\frac{x}{2}, \frac{y}{2}\right)+\phi\left(\frac{y}{2}, \frac{x}{2}\right)+\phi\left(\frac{x}{2}, \frac{x}{2}\right)+\phi\left(\frac{y}{2}, \frac{y}{2}\right)
$$


for all $x, y \in X$. Now,

$$
\begin{aligned}
& N\left(2 f\left(\frac{x+y}{2}\right)-f(x)-f(y), t \phi_{1}(x, y)\right) \\
& \geq N\left(f\left(\frac{x+y}{2}\right)-g\left(\frac{x}{2}\right)-h\left(\frac{y}{2}\right), t \phi\left(\frac{x}{2}, \frac{y}{2}\right)\right) * \\
& N\left(f\left(\frac{x+y}{2}\right)-g\left(\frac{y}{2}\right)-h\left(\frac{x}{2}\right), t \phi\left(\frac{y}{2}, \frac{x}{2}\right)\right) * \\
& N\left(f(x)-g\left(\frac{x}{2}\right)-h\left(\frac{x}{2}\right), t \phi\left(\frac{x}{2}, \frac{x}{2}\right)\right) * \\
& N\left(f(y)-g\left(\frac{y}{2}\right)-h\left(\frac{y}{2}\right), t \phi\left(\frac{y}{2}, \frac{y}{2}\right)\right) \\
& \geq 1-\epsilon \quad[\text { by }(3.5)]
\end{aligned}
$$

for all $x, y \in X$ and $t \geq t_{0}$.

Define a function $F: X \rightarrow Y$ by $F(x)=f(x)-f(0)$. Clearly $F$ satisfies (3.6) and $F(0)=0$. Putting $y=-x$ in (3.6), we get

$$
N\left(-F(x)-F(-x), t \phi_{1}(x,-x)\right) \geq 1-\epsilon
$$

for all $x \in X$ and $t \geq t_{0}$.

Replacing $x$ and $y$ by $-x$ and $3 x$ respectively in (3.6), we get

$$
N\left(2 F(x)-F(-x)-F(3 x), t \phi_{1}(-x, 3 x)\right) \geq 1-\epsilon
$$

for all $x \in X$ and $t \geq t_{0}$. Now,

$$
\begin{aligned}
& N\left(F(x)-3^{-1} F(3 x), t 3^{-1}\left(\phi_{1}(x,-x)+\phi_{1}(-x, 3 x)\right)\right) \\
\geq & N\left(2 F(x)-F(-x)-F(3 x), t \phi_{1}(-x, 3 x)\right) * \\
& N\left(-F(x)-F(-x), t \phi_{1}(x,-x)\right) \\
\geq & 1-\epsilon \quad[\text { by }(3.7),(3.8)]
\end{aligned}
$$

for all $x \in X$ and $t \geq t_{0}$.

Now we show for any positive integer $n$ that

$$
\begin{aligned}
& N\left(3^{-n} F\left(3^{n} x\right)-F(x),\right. \\
& \quad t \sum_{i=0}^{n-1} 3^{-i-1}\left(\phi_{1}\left(3^{i} x, 3^{i}(-x)\right)+\phi_{1}\left(3^{i}(-x), 3^{i}(3 x)\right)\right) \geq 1-\epsilon
\end{aligned}
$$

for all $x \in X$ and $t \geq t_{0}$.

(3.9) shows that (3.10) is true for $n=1$. Let (3.10) be true for $n=k$. Now,

$$
\begin{aligned}
& N\left(3^{-k-1} F\left(3^{k+1} x\right)-F(x),\right. \\
& \left.t \sum_{i=0}^{k} 3^{-i-1}\left(\phi_{1}\left(3^{i} x, 3^{i}(-x)\right)+\phi_{1}\left(3^{i}(-x), 3^{i}(3 x)\right)\right)\right) \geq
\end{aligned}
$$




$$
\begin{aligned}
& \geq N\left(3^{-k-1} F\left(3^{k+1} x\right)-3^{-k} F\left(3^{k} x\right),\right. \\
& \left.t 3^{-k-1}\left(\phi_{1}\left(3^{k} x, 3^{k}(-x)\right)+\phi_{1}\left(3^{k}(-x), 3^{k}(3 x)\right)\right)\right) * \\
& N\left(3^{-k} F\left(3^{k} x\right)-F(x),\right. \\
& \left.t \sum_{i=0}^{k-1} 3^{-i-1}\left(\phi_{1}\left(3^{i} x, 3^{i}(-x)\right)+\phi_{1}\left(3^{i}(-x), 3^{i}(3 x)\right)\right)\right) \\
& \geq 1-\epsilon \quad[\text { by }(3.9)] .
\end{aligned}
$$

This completes the proof of (3.10).

Putting $t=t_{0}$, replacing $n$ by $p$ and $x$ by $3^{n} x$ in $(3.10)$, we get

$$
\begin{gathered}
N\left(3^{-p} F\left(3^{n+p} x\right)-F\left(3^{n} x\right), t_{0} \sum_{i=0}^{p-1} 3^{-i-1}\left(\phi_{1}\left(3^{n+i} x, 3^{i}\left(-3^{n} x\right)\right)+\right.\right. \\
\left.\left.\phi_{1}\left(3^{i}\left(-3^{n} x\right), 3^{i}\left(3^{n+1} x\right)\right)\right)\right) \geq 1-\epsilon
\end{gathered}
$$

for all $x \in X, n \geq 0, p>0$. Again,

$$
\begin{aligned}
& \sum_{i=0}^{p-1} 3^{-i-1}\left(\phi_{1}\left(3^{n+i} x, 3^{i}\left(-3^{n} x\right)\right)+\phi_{1}\left(3^{i}\left(-3^{n} x\right), 3^{i}\left(3^{n+1} x\right)\right)\right) \\
= & \sum_{i=n}^{n+p-1} 3^{n-i-1}\left(\phi_{1}\left(3^{i} x, 3^{i}(-x)\right)+\phi_{1}\left(3^{i}(-x), 3^{i}(3 x)\right)\right) .
\end{aligned}
$$

Since $\sum_{i=0}^{\infty} \frac{\phi\left(3^{i} x, 3^{i} y\right)}{3^{i}}$ converges for all $x, y \in X$, for a given $\delta>0$, there exists $n_{0} \in \mathbb{N}$ such that

$$
\frac{t_{0}}{3} \sum_{i=n}^{n+p-1} 3^{-i}\left(\phi_{1}\left(3^{i} x, 3^{i}(-x)\right)+\phi_{1}\left(3^{i}(-x), 3^{i}(3 x)\right)\right)<\delta
$$

for all $x \in X, n \geq n_{0}$ and $p>0$. Now,

$$
\begin{aligned}
& N\left(\frac{F\left(3^{n} x\right)}{3^{n}}-\frac{F\left(3^{n+p} x\right)}{3^{n+p}}, \delta\right) \\
\geq & N\left(F\left(3^{n} x\right)-3^{-p} F\left(3^{n+p} x\right), t_{0} \sum_{i=0}^{p-1} 3^{-i-1}\left(\phi_{1}\left(3^{n+i} x, 3^{i}\left(-3^{n} x\right)\right)+\right.\right. \\
& \left.\left.\phi_{1}\left(3^{i}\left(-3^{n} x\right), 3^{i}\left(3^{n+1} x\right)\right)\right)\right) \quad[\text { by }(3.12),(3.13)] \\
\geq & 1-\epsilon \quad[\text { by }(3.11)]
\end{aligned}
$$

for all $x \in X, n \geq n_{0}, p>0$.

This shows that the sequence $\left\{3^{-n} F\left(3^{n} x\right)\right\}$ is a Cauchy sequence in $Y$. Since $Y$ is a fuzzy Banach space, the sequence $\left\{3^{-n} F\left(3^{n} x\right)\right\}$ converges to 
some $A(x) \in Y$. So we can define a function $A: X \rightarrow Y$ by

$$
A(x):=N-\lim _{n \rightarrow \infty} 3^{-n} F\left(3^{n} x\right)=N-\lim _{n \rightarrow \infty} 3^{-n} f\left(3^{n} x\right) .
$$

Replacing $x$ and $y$ by $3^{n} x$ in (3.5), we get

(3.15) $N\left(3^{-n} f\left(3^{n} 2 x\right)-3^{-n} g\left(3^{n} x\right)-3^{-n} h\left(3^{n} x\right), 3^{-n} t \phi\left(3^{n} x, 3^{n} x\right)\right) \geq 1-\epsilon$

for all $x \in X, t \geq t_{0}$. Since $\lim _{n \rightarrow \infty} 3^{-n} \phi\left(3^{n} x, 3^{n} x\right)=0$, therefore for fixed $t>0$ and $0<\epsilon<1$, there exists $n_{0} \in \mathbb{N}$ such that

$$
3^{-n} t_{0} \phi\left(3^{n} x, 3^{n} x\right)<\frac{t}{2}
$$

for all $x \in X, n \geq n_{0}$. Now,

$$
\begin{aligned}
& N\left(3^{-n} g\left(3^{n} x\right)+3^{-n} h\left(3^{n} x\right)-A(2 x), t\right) \\
\geq & N\left(3^{-n} f\left(3^{n} 2 x\right)-3^{-n} g\left(3^{n} x\right)-3^{-n} h\left(3^{n} x\right), 3^{-n} t_{0} \phi\left(3^{n} x, 3^{n} x\right)\right) * \\
& N\left(3^{-n} f\left(3^{n} 2 x\right)-A(2 x), \frac{t}{2}\right) \quad[\text { by (3.16)]. }
\end{aligned}
$$

The first term $\geq 1-\epsilon$ by (3.15) and last term tends to 1 as $n \rightarrow \infty$. Thus

$$
\lim _{n \rightarrow \infty} N\left(3^{-n} g\left(3^{n} x\right)+3^{-n} h\left(3^{n} x\right)-A(2 x), t\right)=1
$$

for all $x \in X, t>0$. Hence for all $x \in X$

$$
N-\lim _{n \rightarrow \infty}\left(3^{-n} g\left(3^{n} x\right)+3^{-n} h\left(3^{n} x\right)\right)=A(2 x) .
$$

Again from definition of $A$ we get

$$
\begin{gathered}
A\left(3^{n} x\right)=3^{n} A(x), A(0)=0, \\
\lim _{n \rightarrow \infty} N\left(A(x)-3^{-n} f\left(3^{n} x\right), t\right)=1
\end{gathered}
$$

for all $t>0, x \in X$. Since $\lim _{n \rightarrow \infty} 3^{-n} \phi\left(3^{n} x, 3^{n} y\right)=0$ for all $x, y \in X$, therefore for fixed $t>0$ there exists $n_{1} \in \mathbb{N}$ such that

$$
3^{-n} t_{0} \phi_{1}\left(3^{n+1} x, 3^{n} x\right)<\frac{t}{4}
$$

for all $x \in X, n \geq n_{1}$. Replacing $x$ and $y$ by $3^{n+1} x$ and $3^{n} x$ respectively in (3.6) and for $t=t_{0}$, we get

$$
N\left(2 f\left(3^{n} 2 x\right)-f\left(3^{n+1} x\right)-f\left(3^{n} x\right), t_{0} \phi_{1}\left(3^{n+1} x, 3^{n} x\right)\right) \geq 1-\epsilon
$$

for all $x \in X$. Now,

$$
\begin{aligned}
& N(2 A(2 x)-4 A(x), t) \geq N\left(A(2 x)-3^{-n} f\left(3^{n} 2 x\right), \frac{t}{8}\right) * \\
& N\left(A(3 x)-3^{-n} f\left(3^{n+1} x\right), \frac{t}{4}\right) * N\left(A(x)-3^{-n} f\left(3^{n} x\right), \frac{t}{4}\right) * \\
& N\left(2 f\left(3^{n} 2 x\right)-f\left(3^{n+1} x\right)-f\left(3^{n} x\right), t_{0} \phi_{1}\left(3^{n+1} x, 3^{n} x\right)\right) \quad \text { [by (3.18), (3.20)] }
\end{aligned}
$$


From (3.19) and (3.21), we see that first three terms on RHS tend to 1 as $n \rightarrow \infty$ and last term $\geq 1-\epsilon$. Therefore $N(2 A(2 x)-4 A(x), t)=1$ for all $t>0$. Thus for all $x \in X$

$$
A(2 x)=2 A(x)
$$

Since $\lim _{n \rightarrow \infty} 3^{-n} \phi\left(3^{n} x, 3^{n} y\right)=0$ for all $x, y \in X$, therefore for fixed $t>0$ there exists $n_{2} \in \mathbb{N}$ such that

$$
3^{-n} t_{0} \phi_{1}\left(3^{n} x, 3^{n} y\right)<\frac{t}{4}
$$

for all $n \geq n_{2}$. Replacing $x$ and $y$ by $3^{n} x$ and $3^{n} y$ respectively in (3.6) and for $t=t_{0}$, we get

$$
N\left(2 f\left(\frac{3^{n} x+3^{n} y}{2}\right)-f\left(3^{n} x\right)-f\left(3^{n} y\right), t_{0} \phi_{1}\left(3^{n} x, 3^{n} y\right)\right) \geq 1-\epsilon
$$

for all $x, y \in X$. Now,

$$
\begin{gathered}
N(A(x+y)-A(x)-A(y), t) \geq \\
N\left(A\left(\frac{x+y}{2}\right)-3^{-n} f\left(3^{n}\left(\frac{x+y}{2}\right)\right), \frac{t}{8}\right) * \\
N\left(A(x)-3^{-n} f\left(3^{n} x\right), \frac{t}{4}\right) * N\left(A(y)-3^{-n} f\left(3^{n} y\right), \frac{t}{4}\right) * \\
N\left(2 f\left(\frac{3^{n} x+3^{n} y}{2}\right)-f\left(3^{n} x\right)-f\left(3^{n} y\right), t_{0} \phi_{1}\left(3^{n} x, 3^{n} y\right)\right) \quad[\text { by }(3.22),(3.23)] .
\end{gathered}
$$

From (3.19) and (3.24), we see that first three terms on RHS tend to 1 as $n \rightarrow \infty$ and last term $\geq 1-\epsilon$. Therefore $N(A(x+y)-A(x)-A(y), t)=1$ for all $t>0$ that is, $A(x+y)=A(x)+A(y)$ for all $x, y \in X$.

Let (3.3) hold for some $\delta>0, \alpha>0$. Then by similar approach as in the beginning of proof we can deduce from (3.3) that

$$
\begin{aligned}
& N\left(3^{-n} F\left(3^{n} x\right)-F(x),\right. \\
& \left.\quad \delta \sum_{i=0}^{n-1} 3^{-i-1}\left(\phi_{1}\left(3^{i} x, 3^{i}(-x)\right)+\phi_{1}\left(3^{i}(-x), 3^{i}(3 x)\right)\right)\right) \geq \alpha
\end{aligned}
$$

for all $x \in X, t \geq t_{0}, n \in \mathbb{N}$. Now for $t>0$

$$
N\left(F(x)-A(X), \delta \sum_{i=0}^{n-1} 3^{-i-1}\left(\phi_{1}\left(3^{i} x, 3^{i}(-x)\right)+\phi_{1}\left(3^{i}(-x), 3^{i}(3 x)\right)\right)+t\right) \geq
$$




$$
\begin{aligned}
& N\left(3^{-n} F\left(3^{n} x\right)-F(x), \delta \sum_{i=0}^{n-1} 3^{-i-1}\left(\phi_{1}\left(3^{i} x, 3^{i}(-x)\right)+\phi_{1}\left(3^{i}(-x), 3^{i}(3 x)\right)\right)\right) * \\
& N\left(A(x)-3^{-n} F\left(3^{n} x\right), t\right) .
\end{aligned}
$$

Taking limit as $n \rightarrow \infty$, we get from (3.14) and (3.25)

$N\left(F(x)-A(X), \delta \sum_{i=0}^{\infty} 3^{-i-1}\left(\phi_{1}\left(3^{i} x, 3^{i}(-x)\right)+\phi_{1}\left(3^{i}(-x), 3^{i}(3 x)\right)\right)+t\right) \geq \alpha$.

Because of continuity of $N(x, \cdot)$ and taking limit as $t \rightarrow 0$, we get

$$
N\left(F(x)-A(X), \delta \sum_{i=0}^{\infty} 3^{-i-1}\left(\phi_{1}\left(3^{i} x, 3^{i}(-x)\right)+\phi_{1}\left(3^{i}(-x), 3^{i}(3 x)\right)\right)\right) \geq \alpha,
$$

it proves the result (3.4).

To prove the uniqueness of $A$ let us assume that $A^{\prime}$ be another mapping satisfying (3.2) and (3.4). Then for a given $\epsilon>0$, we can find some $t_{0}>0$ such that

$$
\begin{aligned}
& N(f(x)-A(X)-f(0) \\
& \left.\quad t \sum_{i=0}^{\infty} 3^{-i-1}\left(\phi_{1}\left(3^{i} x, 3^{i}(-x)\right)+\phi_{1}\left(3^{i}(-x), 3^{i}(3 x)\right)\right)\right) \geq 1-\epsilon \\
& N\left(f(x)-A^{\prime}(X)-f(0)\right. \\
& \left.\quad t \sum_{i=0}^{\infty} 3^{-i-1}\left(\phi_{1}\left(3^{i} x, 3^{i}(-x)\right)+\phi_{1}\left(3^{i}(-x), 3^{i}(3 x)\right)\right)\right) \geq 1-\epsilon
\end{aligned}
$$

for all $x \in X, t \geq t_{0}$. Since $\sum_{i=0}^{\infty} \frac{\phi\left(3^{i} x, 3^{i} y\right)}{3^{i}}$ converges for all $x, y \in X$, therefore for a fixed $c>0$ there exists $n_{3} \in \mathbb{N}$ such that

$$
\frac{t_{0}}{3} \sum_{i=n}^{\infty} 3^{-i}\left(\phi_{1}\left(3^{i} x, 3^{i}(-x)\right)+\phi_{1}\left(3^{i}(-x), 3^{i}(3 x)\right)\right)<\frac{c}{2}
$$

for all $n \geq n_{3}$. Again,

$$
\begin{aligned}
& \sum_{i=n}^{\infty} 3^{n-i-1}\left(\phi_{1}\left(3^{i} x, 3^{i}(-x)\right)+\phi_{1}\left(3^{i}(-x), 3^{i}(3 x)\right)\right) \\
= & \sum_{i=0}^{\infty} 3^{-i-1}\left(\phi_{1}\left(3^{n+i} x, 3^{n+i}(-x)\right)+\phi_{1}\left(3^{n+i}(-x), 3^{n+i}(3 x)\right)\right) .
\end{aligned}
$$

Now, for $c>0$

$$
N\left(A(x)-A^{\prime}(x), c\right) \geq
$$




$$
\begin{aligned}
& N\left(A\left(3^{n} x\right)-f\left(3^{n} x\right)+f(0)\right. \\
& \left.\quad t_{0} \sum_{i=0}^{\infty} 3^{-i-1}\left(\phi_{1}\left(3^{n+i} x, 3^{n+i}(-x)\right)+\phi_{1}\left(3^{n+i}(-x), 3^{n+i}(3 x)\right)\right)\right) * \\
& N\left(A^{\prime}\left(3^{n} x\right)-f\left(3^{n} x\right)+f(0), t_{0} \sum_{i=0}^{\infty} 3^{-i-1}\left(\phi_{1}\left(3^{n+i} x, 3^{n+i}(-x)\right)+\right.\right. \\
& \left.\left.\phi_{1}\left(3^{n+i}(-x), 3^{n+i}(3 x)\right)\right)\right) \quad[\text { by }(3.18),(3.28),(3.29)]
\end{aligned}
$$

$\geq 1-\epsilon \quad[$ by $(3.26),(3.27)]$.

It implies that $A(x)=A^{\prime}(x)$ for all $x \in X$. This proves that $A$ is unique.

Replacing $x$ and $y$ by $3^{n+1} x$ and $3^{n} x$ respectively in (3.5), we get

$$
\begin{aligned}
& N\left(3^{-n} f\left(3^{n+1} x+3^{n} x\right)-3^{-n} g\left(3^{n+1} x\right)-\right. \\
& \left.3^{-n} h\left(3^{n} x\right), 3^{-n} t \phi\left(3^{n+1} x, 3^{n} x\right)\right) \geq 1-\epsilon
\end{aligned}
$$

for all $x \in X, n \geq 0, t \geq t_{0}$. Again, replacing $x$ and $y$ by $3^{n} x$ and $3^{n+1} x$ respectively in $(3.5)$, we get

$$
\begin{aligned}
& N\left(3^{-n} f\left(3^{n} x+3^{n+1} x\right)-3^{-n} g\left(3^{n} x\right)-\right. \\
& \left.\quad 3^{-n} h\left(3^{n+1} x\right), 3^{-n} t \phi\left(3^{n} x, 3^{n+1} x\right)\right) \geq 1-\epsilon
\end{aligned}
$$

for all $x \in X, n \geq 0, t \geq t_{0}$. Since $\lim _{n \rightarrow \infty} 3^{-n} \phi\left(3^{n} x, 3^{n} y\right)=0$ for all $x, y \in X$, therefore for fixed $t>0$, there exists $m \in \mathbb{N}$ such that

$$
3^{-n} t_{0}\left(\phi\left(3^{n+1} x, 3^{n} x\right)+\phi\left(3^{n} x, 3^{n+1} x\right)\right)<t
$$

for all $n \geq m$. Now, by (3.32),

$$
\begin{aligned}
& N\left(3^{-n}\left(\left(g\left(3^{n+1} x\right)-h\left(3^{n+1} x\right)\right)-\left(g\left(3^{n} x\right)-h\left(3^{n} x\right)\right)\right), t\right) \geq \\
& N\left(3^{-n} f\left(3^{n+1} x+3^{n} x\right)-3^{-n} g\left(3^{n+1} x\right)-\right. \\
& \left.\quad 3^{-n} h\left(3^{n} x\right), 3^{-n} t_{0} \phi\left(3^{n+1} x, 3^{n} x\right)\right) * \\
& N\left(3^{-n} f\left(3^{n} x+3^{n+1} x\right)-3^{-n} g\left(3^{n} x\right)-\right. \\
& \left.\quad 3^{-n} h\left(3^{n+1} x\right), 3^{-n} t_{0} \phi\left(3^{n} x, 3^{n+1} x\right)\right) \\
& \geq 1-\epsilon \quad[\text { by }(3.30),(3.31)
\end{aligned}
$$


for all $x \in X, t>0, n \geq m$. Let $c>0$. Then we can find a positive integer $m^{\prime} \geq m$ such that

$$
N\left(3^{-m^{\prime}}\left(g\left(3^{m} x\right)-h\left(3^{m} x\right)\right), c\right) \geq 1-\epsilon .
$$

Now, for all $n \geq m^{\prime}, x \in X$,

$$
\begin{aligned}
& N\left(3^{-n}\left(g\left(3^{n} x\right)-h\left(3^{n} x\right)\right), c\right) \\
\geq & N\left(3^{-m}\left(g\left(3^{n} x\right)-h\left(3^{n} x\right)\right), c\right) \quad[\because n \geq m] \\
\geq & N\left(3^{-m^{\prime}}\left(g\left(3^{m} x\right)-h\left(3^{m} x\right)\right), \frac{c}{3^{m^{\prime}-m}(n-m+1)}\right) * \\
& N\left(3^{-m}\left(\left(g\left(3^{m+1} x\right)-h\left(3^{m+1} x\right)\right)-\left(g\left(3^{m} x\right)-h\left(3^{m} x\right)\right)\right), \frac{c}{n-m+1}\right) * \\
& \vdots \\
& N\left(3^{-n+1}\left(\left(g\left(3^{n} x\right)-h\left(3^{n} x\right)\right)-\left(g\left(3^{n-1} x\right)-h\left(3^{n-1} x\right)\right)\right),\right. \\
\geq & 1-\epsilon \quad[\text { by }(3.33),(3.34)] .
\end{aligned}
$$

Therefore, for all $x \in X$,

$$
N-\lim _{n \rightarrow \infty}\left(3^{-n}\left(g\left(3^{n} x\right)-h\left(3^{n} x\right)\right)\right)=0 .
$$

It implies that for all $x \in X$,

$$
N-\lim _{n \rightarrow \infty} 3^{-n} g\left(3^{n} x\right)=N-\lim _{n \rightarrow \infty} 3^{-n} h\left(3^{n} x\right)=A(x)[\operatorname{by}(3.17),(3.22)] .
$$

This completes the proof of the theorem.

Corollary 3.1. Let $a$ be a fixed real number with $0 \leq a<3$ and $\psi:(a, \infty) \rightarrow \mathbb{R}^{+}$be a function such that for all $t, s>a$

$$
\text { (i) } \psi(t s) \leq \psi(t) \psi(s),(i i) \frac{\psi(3)}{3}<1 .
$$

Let $f, g, h: X \rightarrow Y$ be mappings such that

$$
\lim _{t \rightarrow \infty} N(f(x+y)-g(x)-h(x), t(\psi(\|x\|)+\psi(\|y\|)))=1
$$

for all $x, y$ with $\|x\|,\|y\|>a$. Then there exists a unique mapping $A: X \rightarrow Y$ such that $A(x+y)=A(x)+A(y)$ for all $x, y \in X$ and if for some $\delta>0, \alpha>0$

$$
N(f(x+y)-g(x)-h(y), \delta(\psi(\|x\|)+\psi(\|y\|))) \geq \alpha
$$


for all $x, y \in X$, with $\|x\|,\|y\|>a$, then

$$
N\left(f(x)-A(x)-f(0), \frac{\delta}{3-\psi(3)}\left[12 \psi\left(\left\|\frac{x}{2}\right\|\right)+4 \psi\left(\left\|\frac{3 x}{2}\right\|\right)\right]\right) \geq \alpha
$$

for all $x \in X$ with $\|x\|>2 a$.

Proof. Define $\phi(x, y)=\psi(\|x\|)+\psi(\|y\|)$. Then

$$
\widetilde{\phi}(x, y) \leq \frac{3}{3-\psi(3)}(\psi(\|x\|)+\psi(\|y\|)) .
$$

Therefore

$$
\begin{gathered}
\frac{1}{3}\left[\widetilde{\phi}\left(\frac{x}{2}, \frac{-x}{2}\right)+\widetilde{\phi}\left(\frac{-x}{2}, \frac{x}{2}\right)+\widetilde{\phi}\left(\frac{x}{2}, \frac{x}{2}\right)+2 \widetilde{\phi}\left(\frac{-x}{2}, \frac{-x}{2}\right)+\right. \\
\left.\widetilde{\phi}\left(\frac{-x}{2}, \frac{3 x}{2}\right)+\widetilde{\phi}\left(\frac{3 x}{2}, \frac{-x}{2}\right)+\widetilde{\phi}\left(\frac{3 x}{2}, \frac{3 x}{2}\right)\right] \\
\leq \frac{1}{3-\psi(3)}\left[12 \psi\left(\left\|\frac{x}{2}\right\|\right)+4 \psi\left(\left\|\frac{3 x}{2}\right\|\right)\right] .
\end{gathered}
$$

Corollary 3.2. Let $p<1,0 \leq a<3$ and $f, g, h: X \rightarrow Y$ be mappings such that

$$
\lim _{t \rightarrow \infty} N\left(f(x+y)-g(x)-h(x), t\left(\|x\|^{p}+\|y\|^{p}\right)\right)=1
$$

for all $x, y \in X$ with $\|x\|,\|y\|>a$. Then there exists a unique mapping $A: X \rightarrow Y$ such that $A(x+y)=A(x)+A(y)$ for all $x, y \in X$ and if for some $\delta>0, \alpha>0$

$$
N\left(f(x+y)-g(x)-h(y), \delta\left(\|x\|^{p}+\|y\|^{p}\right)\right) \geq \alpha
$$

for all $x, y \in X$, with $\|x\|,\|y\|>a$, then

$$
N\left(f(x)-A(x)-f(0), \frac{4 \delta\left(3+3^{p}\right)}{2^{p}\left(3-3^{p}\right)}\|x\|^{p}\right) \geq \alpha
$$

for all $x \in X$ with $\|x\|>2 a$.

Proof. Define $\psi:(a, \infty) \rightarrow \mathbb{R}^{+}$by $\psi(t)=t^{p}$. Then

$$
\frac{1}{3-\psi(3)}\left[12 \psi\left(\left\|\frac{x}{2}\right\|\right)+4 \psi\left(\left\|\frac{3 x}{2}\right\|\right)\right]=\frac{4\left(3+3^{p}\right)}{2^{p}\left(3-3^{p}\right)}\|x\|^{p} .
$$

Theorem 3.2. Let $\phi: X^{2} \rightarrow \mathbb{R}^{+}$be a mapping such that $\widetilde{\phi}(x, y):=\sum_{i=0}^{\infty} 3^{i} \phi\left(3^{-i} x, 3^{-i} y\right)<\infty$ for all $x, y \in X$.

Let $f, g, h: X \rightarrow Y$ be mappings such that $\lim _{t \rightarrow \infty} N(f(x+y)-g(x)-h(x), t \phi(x, y))=1$ uniformly on $X^{2}$. Then there exists a unique mapping $A: X \rightarrow Y$ such that $A(x+y)=A(x)+A(y)$ for all $x, y \in X$ and if for some $\delta>0, \alpha>0$

$$
N(f(x+y)-g(x)-h(y), \delta \phi(x, y)) \geq \alpha
$$


for all $x, y \in X$, then

$$
\begin{gathered}
N\left(f(x)-A(x)-f(0), \delta\left[\widetilde{\phi}\left(\frac{x}{6}, \frac{-x}{6}\right)+\widetilde{\phi}\left(\frac{-x}{6}, \frac{x}{6}\right)+\widetilde{\phi}\left(\frac{x}{6}, \frac{x}{6}\right)+\right.\right. \\
\left.\left.2 \widetilde{\phi}\left(\frac{-x}{6}, \frac{-x}{6}\right)+\widetilde{\phi}\left(\frac{-x}{6}, \frac{x}{2}\right)+\widetilde{\phi}\left(\frac{x}{2}, \frac{-x}{6}\right)+\widetilde{\phi}\left(\frac{x}{2}, \frac{x}{2}\right)\right]\right) \geq \alpha
\end{gathered}
$$

for all $x \in X$ and

$$
N-\lim _{n \rightarrow \infty} 3^{n}\left(f\left(3^{-n} x\right)-f(0)\right)=A(x)
$$

$$
N-\lim _{n \rightarrow \infty} 3^{n}\left(g\left(3^{-n} x\right)-g(0)\right)=N-\lim _{n \rightarrow \infty} 3^{n}\left(h\left(3^{-n} x\right)-h(0)\right)=A(x) .
$$

Corollary 3.3. Let a be a fixed real number with $a>3$ and $\psi:(0, a) \rightarrow \mathbb{R}^{+}$ be a function such that for all $0<t, s<a$

$$
\text { (i) } \psi(t s) \geq \psi(t) \psi(s),(i i) \frac{\psi(3)}{3}>1 .
$$

Let $f, g, h: X \rightarrow Y$ be mappings such that

$$
\lim _{t \rightarrow \infty} N(f(x+y)-g(x)-h(x), t(\psi(\|x\|)+\psi(\|y\|)))=1
$$

for all $x, y$ with $\|x\|,\|y\|<a$. Then there exists a unique mapping $A: X \rightarrow Y$ such that $A(x+y)=A(x)+A(y)$ for all $x, y \in X$ and if for some $\delta>0, \alpha>0$

$$
N(f(x+y)-g(x)-h(y), \delta(\psi(\|x\|)+\psi(\|y\|))) \geq \alpha
$$

for all $x, y \in X$, with $\|x\|,\|y\|<a$, then

$$
N\left(f(x)-A(x)-f(0), \frac{\delta \psi(3)}{\psi(3)-3}\left[12 \psi\left(\left\|\frac{x}{6}\right\|\right)+4 \psi\left(\left\|\frac{x}{2}\right\|\right)\right]\right) \geq \alpha
$$

for all $x \in X$ with $\|x\|<a$.

Proof. Define $\phi(x, y)=\psi(\|x\|)+\psi(\|y\|)$. Then

$$
\widetilde{\phi}(x, y) \leq \frac{\psi(3)}{\psi(3)-3}(\psi(\|x\|)+\psi(\|y\|)) .
$$

Therefore

$$
\begin{gathered}
\widetilde{\phi}\left(\frac{x}{6}, \frac{-x}{6}\right)+\widetilde{\phi}\left(\frac{-x}{6}, \frac{x}{6}\right)+\widetilde{\phi}\left(\frac{x}{6}, \frac{x}{6}\right)+2 \widetilde{\phi}\left(\frac{-x}{6}, \frac{-x}{6}\right)+ \\
\widetilde{\phi}\left(\frac{-x}{6}, \frac{x}{2}\right)+\widetilde{\phi}\left(\frac{x}{2}, \frac{-x}{6}\right)+\widetilde{\phi}\left(\frac{x}{2}, \frac{x}{2}\right) \\
\leq \frac{\psi(3)}{\psi(3)-3}\left[12 \psi\left(\left\|\frac{x}{6}\right\|\right)+4 \psi\left(\left\|\frac{x}{2}\right\|\right)\right] .
\end{gathered}
$$


Corollary 3.4. Let $p>1, a>3$ and $f, g, h: X \rightarrow Y$ be mappings such that

$$
\lim _{t \rightarrow \infty} N\left(f(x+y)-g(x)-h(x), t\left(\|x\|^{p}+\|y\|^{p}\right)\right)=1
$$

for all $x, y \in X$ with $0 \leq\|x\|,\|y\|<a$. Then there exists a unique mapping $A: X \rightarrow Y$ such that $A(x+y)=A(x)+A(y)$ for all $x, y \in X$ and if for some $\delta>0, \alpha>0$

$$
N\left(f(x+y)-g(x)-h(y), \delta\left(\|x\|^{p}+\|y\|^{p}\right)\right) \geq \alpha
$$

for all $x, y \in X$, with $0 \leq\|x\|,\|y\|<a$, then

$$
N\left(f(x)-A(x)-f(0), \frac{4 \delta\left(3^{p}+3\right)}{2^{p}\left(3^{p}-3\right)}\|x\|^{p}\right) \geq \alpha
$$

for all $x \in X$ with $0<\|x\|<a$.

Proof. Define $\psi:(0, a) \rightarrow \mathbb{R}^{+}$by $\psi(t)=t^{p}$. Then

$$
\frac{\psi(3)}{\psi(3)-3}\left[12 \psi\left(\left\|\frac{x}{6}\right\|\right)+4 \psi\left(\left\|\frac{x}{2}\right\|\right)\right]=\frac{4\left(3^{p}+3\right)}{2^{p}\left(3^{p}-3\right)}\|x\|^{p} .
$$

\section{Conclusion}

In this paper the generalized Hyers-Ulam-Rassias stability of the functional equation $f(x+y)=g(x)+h(y)$ has been studied in fuzzy Banach spaces. What could be the general solution of such functional equation and what are the properties of the general solution of this equation, it should be studied in future. Instead of crisp functional equation, if we consider fuzzy functional equation, how can we study the corresponding Hyers-Ulam stability property.

Acknowledgement: The authors are extremely grateful to the reviewers for their suggestions to improve our paper.

\section{REFERENCES}

[1] A. George and P. Veeramani, On Some result in fuzzy metric spaces, Fuzzy Sets and Systems, 64 (1994), 395-399.

[2] A. K. Katsaras, Fuzzy Topological Vector Space, Fuzzt Sets and System, 12 (1984), $143-154$.

[3] A. K. Mirmostafaee, M. S. Moslehian, Fuzzy versions of HyersÜUlamÜRassias theorem, Fuzzy Sets Syst., 159 (2008), 720729.

[4] B. Schweizer, A. Sklar, Statistical metric space, Pacific journal of mathematics, 10 (1960) 314-334.

[5] C. Park, Fuzzy stability of a functional equation associated with inner product space, Fuzzt set and system, 160 (2009), 16321642.

[6] D. H. Hyers, On the stability of the linear functional equation, Proc. Nat. Acad. Sci. U.S.A., 27 (1941), 222-224.

[7] F. Skof, Proprieta locali e approssimazione di opratori, Rend. Sem. Mat. Fis. Milano, 53 (1983), 113-129.

[8] L. A. Zadeh, Fuzzy sets, Information and control, 8 (1965), 338-353. 
[9] N. Chandra Kayal, P. Mondal and T. K. Samanta The Generalized Hyers - Ulam Rassias Stability of a Quadratic Functional Equation in Fuzzy Banach Spaces, Journal of New Results in Science, 1 (5) (2014), 83-95.

[10] P. Gavruta, A generalization of the Hyers-Ulam-Rassias Stability of approximately additive mappings, J. Math. Anal. appl., 184 (1994), 431-436.

[11] P. W. Cholewa, Remarks on the stability of functional equations, Aequationes Math., 27 (1984), 76-86.

[12] S. C. Cheng and J. N. Moderson, Fuzzy Linear Operator and Fuzzy Normed Linear Space, Bull. Cal.Math. Soc., 86 (1994), 429-438.

[13] S. Czerwik, On the stability of the quadratic mappings in normed spaces, Abh. Math. Sem. Univ. Hamburg, 62 (1992), 59-64.

[14] S. M. Ulam, Problems in Modern Mathematics, Chapter VI, Science Editions, Wiley, New York, 1960.

[15] T. Aoki, On the Stability of Linear Transformation in Banach Spaces, J. Math. Soc. Japan, 2 (1950), 64-66.

[16] Th. M. Rassias, On the stability of the linear additive mapping in Banach space, Proc. Amer. Mathematical Society, 72(2) (1978), 297-300.

[17] T. K. Samanta and Iqbal H. Jebril, Finite dimentional intuitionistic fuzzy normed linear space, Int. J. Open Problems Compt. Math., Vol.2, No.4, (2009), 574-591.

[18] T. K. Samanta, P. Mondal and N. Chandra Kayal, The generalized Hyers-UlamRassias stability of a quadratic functional equation in fuzzy Banach spaces, Annals of Fuzzy Mathematics and Informatics, Vol. 6, No. 2, (2013), 285294.

[19] T. K. Samanta, N. Chandra Kayal and P. Mondal, The Stability of a General Quadratic Functional Equation in Fuzzy Banach Space, Journal of Hyperstructures, Vol.1, No.2, (2012), 71-87.

\author{
Nabin Chandra Kayal \\ Department of Mathematics \\ Moula Netaji Vidyalaya, \\ Moula, Howrah, 711312 West Bengal \\ INDIA \\ E-mail address: kayalnabin82@gmail.com
}

Pratap Mondal

Department of Mathematics

Orphuli Uday Chand Memorial Institute

Orphuli, Bagnan, Howrah, 711303 West Bengal

INDIA

E-mail address: pratapmondal111@gmail.com

\title{
T.K. SAmanta
}

Department of Mathematics

Uluberia College

Uluberia, Howrah, 711315 West Bengal

INDIA

E-mail address: mumpu_tapas5@yahoo.co.in 